\title{
A Monte Carlo Study of Restricted Diffusion: Implications for Diffusion MRI of Prostate Cancer
}

\section{Nima Gilani ${ }^{1}$, MS, Paul Malcolm², MBBS, Glyn Johnson ${ }^{1}$, PhD. \\ ${ }^{1}$ Norwich Medical School, University of East Anglia \\ ${ }^{2}$ Norfolk and Norwich University Hospital}

Corresponding author: Glyn Johnson

\author{
Bob Champion Research and Educational Building \\ James Watson Road \\ University of East Anglia \\ Norwich Research Park \\ Norwich NR4 7UQ \\ UK \\ Telephone: + 44 (1603) 593682 \\ glyn.johnson@uea.ac.uk
}

Word Count: 3980

1 Table and 4 Figure Parts 


\begin{abstract}
Purpose: Diffusion MRI is frequently used to assess prostate cancer. The prostate consists of cellular tissue surrounding fluid filled ducts. Here, the diffusion properties of the ductal fluid alone were studied. Monte Carlo simulations were used to investigate ductal residence times to determine whether ducts can be regarded as forming a separate compartment and whether ductal radius could determine the ADC of the ductal fluid.
\end{abstract}

Methods: Random walks were simulated in cavities. Average residence times were estimated for permeable cavities. Signal reductions resulting from application of a Stejskal-Tanner pulse sequence were calculated in impermeable cavities. Simulations were repeated for cavities of different radii and different diffusion times.

Results: Residence times are at least comparable with diffusion times even in relatively high grade tumours. ADCs asymptotically approach theoretical limiting values. At large radii and short diffusion times, ADCs are similar to free diffusion. At small radii and long diffusion times, ADCs are reduced towards zero, and kurtosis approaches a value of -1.2.

Conclusions: Restricted diffusion in cavities of similar sizes to prostate ducts may reduce ductal ADCs. This may contribute to reductions in total ADC seen in prostate cancer.

Keywords: Prostate Cancer, Diffusion MRI, Monte Carlo, Non-Gaussian Restricted Diffusion, Negative Kurtosis 


\section{Introduction}

The use of quantitative diffusion MRI (Magnetic Resonance Imaging) for assessment of prostate cancer has been investigated by many groups [1-10]. Interpretation of these measurements is complicated by the structure of prostate tissue which consists of fluid filled ducts surrounded by epithelial and stromal cells. The radius of the ductal lumen seen in histological sections of the normal prostate is $\sim 300 \mu \mathrm{m}[11,12]$ which is at least an order of magnitude greater than one dimensional Einstein diffusion distance of $\sim 20 \mu \mathrm{m}$ (assuming a diffusion time of $80 \mathrm{~ms}$, typical of clinical scanners, and diffusion coefficient of $3 \mu \mathrm{m}^{2} \mathrm{~ms}^{-1}$, similar to that of water $[13,14])$. The residence time of a water molecule within ducts in normal prostate is therefore likely to be long compared with the diffusion time. Consequently, the ductal and cellular (i.e., epithelial plus stromal) spins will effectively reside in separate compartments and generate separate signals each with its own characteristic parameters. Although there are no direct experimental demonstrations of separate ductal and cellular compartments, the hypothesis is consistent with the observation of biexponential diffusion $[3,15]$ and $\mathrm{T}_{2}$ relaxation $[16,17]$. Furthermore, the ADC of the fast diffusing $(2-3$ $\left.\mu \mathrm{m}^{2} \mathrm{~mm}^{-1}[3,15]\right)$ and long $\mathrm{T} 2(\sim 500 \mathrm{~ms}[16,18])$ components in the prostate are typical of fluids and much greater than values found in cellular tissue (ADC $\sim 0.7 \mu \mathrm{m}^{2} \mathrm{~mm}^{-1}$ [19] and T2 $\sim 80 \mathrm{~ms}$ [20] in the brain, for example). (In the following we use 'ADC' to refer to any measured approximation of a diffusion coefficient, regardless of the equation used for fitting.) We therefore hypothesize that the biexponential signal components seen in DWI (and $\mathrm{T} 2$ relaxometry) of the prostate arise primarily from the glandular fluid and the cellular compartments.

It is known from both hyperpolarized ${ }^{3} \mathrm{He}$ studies in the lungs [21] and studies in porous media [22-24], that measured diffusion coefficients can depend strongly on the size of the cavity in which the studied molecule resides. Furthermore, Jensen et al. [25, 26] have given several examples where hindrance, compartmentalization and restriction lead to nonGaussian behaviour of diffusion. Analytical formulations of restricted diffusion inside cylindrical and spherical pores have previously been presented [27-30]. However, although the non-Gaussian nature of restricted diffusion was recognized in these studies, diffusion decays were assumed to be simple exponentials for simplicity. It is not clear that analytical solutions that include the effects of non-Gaussian diffusion are possible. Monte Carlo simulation is an alternative to analytical methods that is both easily extensible to non- 
Gaussian distributions and also to complex geometries. Monte Carlo studies of restricted diffusion in two compartments [31] and in depth analysis of restricted diffusion [32] have already been published. However Monte Carlo simulation of diffusion in single compartments has not been published previously.

This paper primarily considers diffusion within the ductal fluid alone. Although this is only one component of a complex tissue, it is necessary to understand the properties of each component individually before the overall diffusion properties of the prostate can be understood. We wish to answer three questions. 1) What are the residence times of water within the ductal lumen with the reduced radii seen in cancer; and hence, can the ductal fluid be treated as a separate compartment in cancer? 2) Could restricted diffusion within the ductal lumen determine the diffusion coefficient and kurtosis of the ductal fluid? 3) Are changes in ductal diameter that occur in cancer [11, 12, 33] sufficient to alter measured diffusion coefficient and kurtosis of the ductal fluid?

The purpose of this study was therefore threefold.

1. To estimate ductal radius in normal and cancerous prostate from figures obtained from histology texts.

2. To use Monte Carlo simulations of permeable cavities to determine the relationship between permeability, cavity size and residence times; and to establish plausible limits to residence times.

3. If the results of aim two justify the assumption of long residence times, to use Monte Carlo simulates of impermeable cavities to investigate the effect of cavity size on diffusion and kurtosis. The assumption of impermeable cavities greatly simplifies the simulations and has been adopted in previous studies [34].

\section{Theory}

\section{Residence Times}

The ductal lumen can be considered a separate compartment from the surrounding epithelium and stroma if the residence times of water molecules inside are long compared with diffusion times [31]. Residence time is determined by ductal compartment size and geometry and the permeability of its boundaries, usually described in units of $\mu \mathrm{ms}^{-1}$. Regan and Kuchel $[35,36]$ 
and Lee et al. [37] calculated the equivalent probability, $p$, of crossing upon hitting a barrier in random walk simulations:

$$
p=\kappa \sqrt{\frac{6 \Delta t}{D_{\text {free }}}}
$$

where $\kappa$ is permeability, $D_{\text {free }}$ is the diffusion coefficient in the absence of barriers, and $\Delta t$ is the time step of the random walks.

\section{Diffusion}

The average distance moved by a freely diffusing spin in three dimensions is given by the Einstein diffusion equation [38]

$$
\langle r\rangle=\sqrt{6 D_{\text {free }} T_{D}}
$$

where $D_{\text {free }}$ is again the unrestricted diffusion coefficient or diffusion coefficient in very small times compared to the boundaries and $T_{D}$ is the time over which diffusion occurs. When the spin is constrained within a small cavity the apparent diffusion coefficient, $D$, will be reduced. From Eq. (2) it is clear that $D$ is a function of the ratio, $\alpha$,

$$
\alpha=\frac{r_{d}^{2}}{T_{D}}
$$

where $r_{d}$ is the radius of the cavity. In other words, when diffusion times are long, spins diffuse further and reach the walls of relatively large cavities. Conversely, spins reach the walls of small cavities in short diffusion times.

We expect the following asymptotic behavior of $D$

$$
\begin{aligned}
& \lim _{\alpha \rightarrow 0} D=\frac{\alpha}{6} \\
& \lim _{\alpha \rightarrow \infty} D=D_{\text {free }}
\end{aligned}
$$

i.e., for very small $\alpha$, all spins traverse the cavity many times and ADC is that for $\langle r\rangle=r_{d}$; for very large $\alpha$, most spins never reach the walls of the cavity and diffusion is effectively free. 
When diffusion is restricted or the diffusion signal arises from more than one compartment, the probability density function, $\mathrm{P}(r)$, describing diffusional displacement becomes nonGaussian. The excess kurtosis, $K$, is the normalized the fourth moment of $\mathrm{P}[25,26]$ :

$$
K=\frac{\int r^{4} P(r) d r}{\left(\int r^{2} P(r) d r\right)^{2}}-3
$$

where $r$ is displacement and the subtraction of 3 is conventionally applied to ensure that the kurtosis of a Gaussian is zero. For non-Gaussian P, $K$ becomes non-zero. Kurtosis is a complex function that depends on tissue complexity and microgeometry, the disparity in diffusion coefficients between different tissue components and the degree of restriction [25]. In this study, kurtosis is only a function of the degree of restriction in the simulated component. When $\alpha$ is large, $\mathrm{P}$ is nearly Gaussian and $K$ is close to zero. Conversely, when $\alpha$ is small, each molecule traverses the cavity many times, $\mathrm{P}$ becomes increasingly platykurtic (boxy) as it approaches a boxcar function for which it is simple to show from Eq. (5) that $K=$ -1.2 . Accordingly we expect the following asymptotic behavior of $K$ :

$$
\begin{aligned}
& \lim _{\alpha \rightarrow 0} K=-1.2 \\
& \lim _{\alpha \rightarrow \infty} K=0.0 .
\end{aligned}
$$

\section{Methods}

\section{Ductal Radius}

From histological studies $[11,12,33]$ it is clear that ductal radius varies widely and differs in different grades of cancerous prostate. However, to our knowledge no estimates of radius have been published. We therefore measured radii in histology sections obtained from two different references $[11,12]$. The histology sections were scanned and loaded into ImageJ V1.48 [39]. The lumen of individual ducts were segmented and the area measured. Radii were calculated assuming circular lumen (i.e., $r=\sqrt{A / \pi}$ where $A$ is the lumenal area). A minimum of 20 different measurements were obtained for each figure from each source and the minimum, maximum and mean radii recorded. The process was performed on sections of normal peripheral zone, Gleason grades 3, 4 and 5, and a section with Gleason score 3+4. 


\section{Residence Times}

All simulations were performed in MATLAB Release 2013b (MathWorks, Natick, MA).

Residence times were estimated from simulations similar of those of Regan and Kuchel [36] and Fieremans et al. [31]. Spins were placed randomly within spherical cavities and random walks simulated as follows. At each time step, the spin was moved a fixed distance $\Delta \mathbf{r}$ in a random direction. The length of $\Delta \mathbf{r}$ is determined by the unrestricted diffusion coefficient of the fluid,

$$
|\Delta \mathbf{r}|=\sqrt{6 D_{\text {free }} \Delta t}
$$

where $\Delta t$, the step time, is $T_{D}$ divided by $N_{s}$, the number of steps in the simulation. For this study we assumed that $D_{\text {free }}$ was that of free water at $37^{\circ} \mathrm{C}\left(3.08 \mu \mathrm{m}^{2} \mathrm{~ms}^{-1}\right)[13,14] . \Delta t$ was $25 \mu$ s was used giving $|\Delta \mathbf{r}| \approx 0.65 \mu \mathrm{m}$.

If the step $\Delta \mathbf{r}$ took the spin beyond the boundary of the cavity a random number was drawn from a uniform distribution with minimum zero and maximum one. If this value as less than the assigned probability of crossing (Eq. (1)) then the spin was assumed to have exited and the total time to that point recorded. Otherwise, the spin was placed back at its previous position and randomly moved in a different direction at the next step. This approach has previously been shown to give similar results to the alternative of elastic reflection [40].

This procedure was repeated for 1,000,000 spins and the mean of all times recorded as the mean residence time.

The ductal lumen is surrounded by a single layer of epithelial cells with thickness of $\sim 10 \mu \mathrm{m}$. $[11,12]$. Crossing probability (Eq. (1)) requires an estimate of the permeability of this layer which has not be measured as far as we know. However, the permeability of cells walls has been estimated to be anywhere between 6 and $200 \mu \mathrm{ms}^{-1}$ [41-44]. This simulation was therefore performed with a somewhat conservative value, twice the maximum value found in cell walls, $400 \mu \mathrm{ms}^{-1}$. With this permeability, the probability of a spin crossing the cavity wall on hitting it is $8.8 \%$ (Eq. (1)). 


\section{Diffusion}

If residence times are long relative to diffusion times, then semi-permeable cavitites can be approximated by impermeable cavities. This approach has been used previously and greatly simplifies simulations [34]. Moreover, the probability that a spin crosses the boundary at a single collision is less than $10 \%$ even with high values of permeability (see above). Hence, even with permeable barriers diffusion is substantially impeded. We therefore believe the approximation is reasonable.

Six different values of sphere $r_{d}(10,20,30,40,50$, and $60 \mu \mathrm{m})$ and 12 values of $T_{D}(40,50$, $60,64,70,80,90,100,133,150,180$ and $200 \mathrm{~ms}$ ) were simulated to give a total of 27 different values of $\alpha$. This range of values gives a range of $\alpha$ such that predicted values of $D$ range between zero and $D_{\text {free. }}$. Five different values of cylinder $r_{d}(10,20,30,50$ and $90 \mu \mathrm{m})$ and 3 values of $T_{D}(50,80$ and $100 \mathrm{~ms})$ were simulated to give a total of 12 different values of $\alpha$. Cylinder height, $z$, was either two or four times the radius. Some values of $\alpha$ were simulated multiple times with different combinations of $r_{d}$ and $T_{D}$.

Spins were placed randomly in these cavities and 40,000 random walks generated as above for each cavity.

Phase shifts caused by Stejskal-Tanner [45] PGSE gradients and hence the signal were calculated using Hall et al.'s [46] method. At each step, $i$, each spin accumulates a phase $\delta \phi_{i}$ relative to spins at the zero point of the gradients

$$
\delta \varphi_{i}=\gamma \mathbf{G} . \mathbf{r} \Delta t
$$

where $\gamma$ is the gyromagnetic ratio, $\mathbf{r}$ is the position of the spin, $\mathbf{G}$ is the applied gradient and $\Delta t$ is the time of each step. The signal from each spin is equal to the cosine of the final phase

$$
s=\cos \left(\sum_{i=1}^{N_{s}} \delta \varphi_{i}\right)
$$

and total signal is the sum of the signals from all spins. This calculation was performed separately for $\mathrm{x}, \mathrm{y}$ and $\mathrm{z}$ diffusion weighting gradients using the same trajectories.

For the cylinders these calculations were performed in 15 different directions and averaged to nullify out directionality effects. 
Estimates of apparent diffusion coefficient and apparent diffusion kurtosis were obtained from the simulated signals as follows. Signals were simulated for 50 equally distanced $b$ values using the same spin trajectories. The maximum b-value was dependent on $\alpha$ and ranged from around $500 \mathrm{~s} . \mathrm{mm}^{-2}$ for very high $\alpha$ s to around $15000 \mathrm{~s} . \mathrm{mm}^{-2}$ for very low $\alpha \mathrm{s}$. The following expression [25] was then fitted to the signals

$$
S(b)=S(0) e^{-b D+\frac{K D^{2} b^{2}}{6}}
$$

where $D$ and $K$ are the apparent diffusion coefficient and apparent diffusion kurtosis respectively. Three values of $D$ and $K$ were obtained for the $\mathrm{x}, \mathrm{y}$ and $\mathrm{z}$ directions and averaged.

\section{Results}

\section{Ductal Radius}

Radius measurements obtained from the two different sources [11, 12] did not appear to differ substantially so were combined. There was considerable variability, possibly due to sectioning of ducts that are approximately cylindrical at a variety of angles. Nonetheless consistent reductions in diameter with increasing tumour grade are seen. Results are given in Table 1.

\section{Residence Times}

Table 1 gives the estimates of residence times for ducts in tissues of different Gleason grades, assuming a high permeability value of $400 \mu \mathrm{ms}^{-1}$. These times are at least comparable with typical in vivo diffusion times at least up to Gleason score $3+4$. The times are also somewhat greater than intracellular residence times measured for glioma cells (50 ms [47]), bovine optic nerve (62 ms [48]), and red blood cells (10-14 ms [49]).

\section{Diffusion}

Estimated values of $D$ and $K$ derived from the sphere and cylinder simulations are plotted against $\alpha$ in Fig. 1. Asymptotic values of $D$ (Eq. (4)) are also plotted for the sphere as $\alpha$ approaches zero. Results are somewhat noisy due to the limited number of simulations run. However, simulation results agree very well with the expected asymptotes. At large $\alpha$ (i.e., large radii and/or shorter diffusion times), $D$ approaches the free diffusion coefficient and kurtosis approaches zero. Conversely at small $\alpha$ (i.e., small radii and/or long diffusion times) 
$D$ approaches zero and kurtosis approaches a value of -1.2 , equal to that of a uniform distribution. As far as we are aware this is the first suggestion that negative values of kurtosis might be found in tissue. Values are similar for spheres and cylinders, particularly the small cylinders. This might be expected since diffusion distances in two out of the three directions (perpendicular to the axis of the cylinder) will be equal in cylinders and spheres.

Fig. 2 gives a plot of $D / D_{\text {free }}$ and $K$ against Gleason scores using measured radii (Table 1 ). Other parameters were $T_{D}=80 \mathrm{~ms}$ and $D_{\text {free }}=3.08 \mu \mathrm{m}^{2} \mathrm{~ms}^{-1}$.

Finally, to illustrate the effect of negative kurtosis, $\ln \left(S / S_{0}\right)$ is plotted against the dimensionless product $b D$, for three different values of $\alpha$ in Fig. 3. ( $b D$ was chosen as the abscissa to emphasize the increase in relative size of the kurtosis effect at low $\alpha$.) As $\alpha$ approaches zero, diffusion becomes increasing platykurtic and the signal drops relative to Gaussian diffusion.

\section{Discussion and Conclusions}

The biexponential behavior of both $\mathrm{T}_{2}[16,17]$ and diffusion $[3,15]$ in the prostate has been experimentally demonstrated. Although other explanations exist for bi-exponential behavior, one possibility is the existence of two separate compartments with long spin residence times. We have hypothesized that these two compartments correspond to spins within the ductal fluid and the surrounding cellular tissue (stroma plus epithelium). This is consistent with two observations. First, the fast diffusion coefficient and long $T_{2}$ are typical of fluids and much greater than seen in cellular tissues. Second, simple biophysical arguments show that spin residence times in ductal fluid are long in normal prostate because ductal diameter is much greater than diffusion distances. Panagiotaki et al. [50] have attributed biexponential behavior to slow exchange between intra- and extra-cellular water. However, biexponential $\mathrm{T}_{2} \mathrm{~S}$ observed in other tissues are $\sim 10$ and $\sim 80 \mathrm{~ms}$ [20] both much shorter than the long $\mathrm{T}_{2}$ seen in the prostate $(\sim 500 \mathrm{~ms})$. Moreover, the residence times we have estimated for ductal lumen are rather greater that those observed for intracellular water [31, 47, 48]. It therefore seems probable that at least some (if not most) of the observed biexponential behavior should be attributed to separate ductal and cellular compartments.

In normal prostate, ductal radii are an order of magnitude greater than diffusion distances. Ductal and cellular spins must therefore present as different compartments. In cancer, 
however, ductal radii are much reduced and become comparable with diffusion distances at Gleason grade 5. Our simulations suggest that residence times in permeable ducts may also become comparable with diffusion times at about Gleason grade 3 or 4. Nonetheless, biexponentiality is still observed in cancer $[3,16]$. It is quite possible that a second source of compartmentalization becomes dominant in cancer. However, it seems likely that ductal compartmentalization also contributes to the biexponential signals seen in cancer.

The ductal volume in normal prostate has been measured histologically to be about $28 \%$ [51]. The observation of a short $\mathrm{T}_{2}$ signal fraction of $27 \%$ seen by Storas et al. [16] and $40 \%$ seen by Gilani et al. [17] is reasonably consistent with this figure. However, the fast diffusing signal fraction seen by Shinmoto et al. in vivo [3] and by Bourne et al. in fixed samples [52] were $\sim 70 \%$, much greater than would be expected from the histological volume. Some of this difference can be accounted for by greater spin density in ductal fluid relative to cellular tissue - the glandular fluid contains few solids so that water content is $100 \%$ whereas most soft tissues have a water content of about $75 \%[53,54]$. Some of the difference will be due to perfusing spins (the IVIM effect); and some to the effect of non-Gaussian diffusion in either compartment. However, Bourne et al. [52] also observed a fast diffusing component in fixed cellular tissues. Although the measured ADC of this component $\left(1.56 \mu \mathrm{m}^{2} \mathrm{~mm}^{-1}\right)$ was rather lower than that seen in pure ductal lumen $\left(2.2 \mu \mathrm{m}^{2} \mathrm{~mm}^{-1}\right)$ it seems likely that this also contributes to the fast component seen in vivo.

Jensen et al. [25] suggested platykurtic diffusion may occur for restricted diffusion in pores but most previous reports (e.g., [55-60]) have suggested leptokurtic diffusion is the norm. However, these studies considered the net diffusion properties of tissues consisting of multiple tissue components with a variety of different water exchange rates. Results are therefore consistent with Jensen et al.'s [25, 26] finding of overall leptokurtic diffusion in tissues consisting of two exchanging Gaussian compartments. Similarly, Rosenkrantz et al. [9] found apparent leptokurtic diffusion for all the three compartments of the prostate as a whole but did not consider the possibility of biexponential diffusion which will mimic monoexponential diffusion with positive kurtosis over the range of b-values used (maximum $2000 \mathrm{smm}^{-2}$ ). The possibility of restricted, platykurtic diffusion may need to be taken into account in investigations of the net diffusion properties of tissues with different slowly exchanging compartments, e.g., between intra- and extra-cellular water fractions. 
The effect of compartment size on measured diffusion coefficient is recognized in hyperpolarized gas imaging of the lungs [21] where alveolar enlargement in emphysema explains increases in measured diffusion coefficient. The phenomenon is also well known in diffusion measurements in porous media [22-24] where cavity size is often explored using diffusion measurements at multiple values of $T_{D}$. Here we have used Monte Carlo simulations to show that lumenal size may influence the measured diffusion coefficients of ductal fluid in the prostate. This finding is consistent with several recent studies of the prostate both in vivo and in vitro.

First, Shinmoto et al. [3] observed biexponential diffusion in prostate tissue and found fast ADCs of 2.9 and $1.7 \mu \mathrm{m}^{2} \mathrm{~ms}^{-1}$ in healthy prostate and prostate cancer respectively. Their diffusion time is not given but $T_{E}$ was $91 \mathrm{~ms}$, so that $T_{D}$ would be around $80 \mathrm{~ms}$. With this $T_{D}$ an ADC of $1.7 \mu \mathrm{m}^{2} \mathrm{~ms}^{-1}$ corresponds to $r_{d}=35 \mu \mathrm{m}$ (Fig. 1a). This is similar to the ductal radii we measured in tissues with Gleason scores between 7 and 8 (Table 1).

Second, the measured fast diffusion coefficients has been shown to depend on diffusion time in ex vivo prostate samples [61] using either Gaussian or non-Gaussian biexponential fits. This time dependency and the dependency on cavity size are essentially the same phenomena viewed from different perspectives (see Eq. (3)) thus these findings are consistent with those presented here.

Finally, Chatterjee et al. [62] recently showed that the relative volumes of the different prostate compartments is a determining factor in ADC measurements and, furthermore, that correlations between ADC and relative volumes were stronger than with cellularity.

We have used Jensen et al.'s kurtosis framework to describe the non-Gaussian nature of restricted diffusion. Alternative equations, e.g., a stretched exponential [63], could also be used. However, the use of a stretched exponential is an essentially heuristic device with no easily interpreted connection with the underlying biophysics of diffusion. Although kurtosis cannot unambiguously be associated with any specific microstructural feature of the tissue, it does relate directly to the probability density function that describes diffusional motion. For this reason we prefer it as an analysis method.

An association between lumenal radius and diffusion within the ductal fluid, if confirmed, would have a number of interesting implications. First, if $D, K$ and the ductal signal fraction, $f_{d}$ are all well-defined functions of $r_{d}$ then an estimate of any one would allow estimation of 
the others, minimizing the number of $b$ values required for measurement. Furthermore, $f_{d}$ could be estimated from biexponential $T_{2}$ measurements (which can be somewhat easier to make) thus further simplifying measurement. Second, as recently discussed by Bourne [64], given the diffusion time dependency of measurements, it is important that future diffusion studies of the prostate should include this parameter and consequently that scanner manufacturers should include it in sequence specifications. Finally, by measuring biexponential ADCs as a function of diffusion time it might be possible to estimate ductal radius. Since duct size is a key determinant of Gleason grade this might improve the accuracy of cancer diagnosis and grading. Although this could be challenging, Shinmoto et al. [3] have previously measured reductions in the ADC of the fast diffusing component in cancer.

There are a number of limitations to this study. Most obviously, it considers only one component of a complex system and ignores signals arising from the cellular (i.e., stromal plus epithelial) compartment. Development of a full model for the prostate incorporating signals from all compartments, and considering exchange between them, is the subject of ongoing research. Second, we have only considered a system in which the biexponential diffusion behavior of the prostate is due to compartmentization of spins between cellular tissue and ductal lumen. There is no direct evidence for this and other explanations are possible. However, we believe that we have demonstrated that such a system is plausible and largely consistent with the data. Third, simulations of restricted diffusion assumed impermeable spheres. This is an unrealistic approximation that, at first sight, invalidates the assumption that restricted diffusion occurs in the prostate ducts. However, even with high permeability assumed here, the probability of a spin crossing the barrier at an individual collision is less than $10 \%$. Consequently, although diffusion is not strictly restricted, it is heavily impeded and ADC will be reduced as a result. However, the estimates of ADC and $K$ presented here must be regarded as lower limits. Fourth, it would be better to compare our results with tumours well characterized with post-surgical, whole-mount Gleason scores. Similarly it would be better to compare the simulations with diffusion measurements explicitly designed to estimate the full complexity of diffusion in the prostate. Again, this is the subject of ongoing research. Fifth, cylinders or spheres may not provide a realistic representation of lumenal ducts. However, our main objective was to demonstrate that diffusion within structures of size similar to that of the ductal lumen can demonstrate restricted diffusion and thus can determine measured $D_{d}$. Finally, the free diffusion coefficient of ductal fluid may be somewhat less than that of water as assumed here because 
of high protein concentration [65]. This would reduce the effect of changes in ductal radius on diffusion. However, the effect is unlikely to be large.

\section{Conclusion}

Restricted diffusion in cavities of similar sizes to prostate ducts may reduce ductal ADCs. This may contribute to reductions in total ADC seen in prostate cancer. 


\section{Tables}

Table 1. Ductal radius for different Gleason grades and equivalent residence times assuming ductal permeability of $400 \mu \mathrm{ms}^{-1}$.

\begin{tabular}{|l|c|c|}
\hline & $\begin{array}{c}\text { Ductal Radius } \\
\text { Mean } \pm \text { SD (range) } / \mu \mathrm{m}\end{array}$ & Residence Time / ms \\
\hline Healthy PZ & $300 \pm 120(50-500)$ & 2100 \\
\hline Grade 3 & $65 \pm 36(15-110)$ & 130 \\
\hline Score 3+4 & $45 \pm 24(5-110)$ & 70 \\
\hline Grade 4 & $30 \pm 13(5-50)$ & 35 \\
\hline Grade 5 & $20 \pm 8(3-40)$ & 21 \\
\hline
\end{tabular}




\section{Figure Captions}

Figure 1. Diffusion parameters derived by fitting Eq. (10) to simulated signals plotted as a function of $\alpha$. Simulations were performed at six different radii with $T_{D}$ adjusted to provide the required value of $\alpha$. a) D vs. $\alpha$. The dotted line gives the asymptote for $(\alpha / 6)$ from Einstein's formula. b) $K$ vs. $\alpha$.

Figure 2. $D / D_{\text {free }}$ and $\mathrm{K}$ of lumenal fluid predicted for different Gleason scores (normal peripheral zone $=1) . T_{D}=80 \mathrm{~ms}$ and $D_{\text {free }}=3.08 \mu \mathrm{m}^{2} \mathrm{~ms}^{-1}$.

Figure 3. Plot of $\ln \left(S / S_{0}\right)$ vs. dimensionless parameter $b D$ for $\alpha=1,10$ and $\infty$ (i.e., free diffusion) corresponding to $D=0.15,1.44$ and $3.08 \mu \mathrm{m}^{2} \mathrm{~mm}^{-1}$ and $K=-1.1,-0.42$ and 0 . 


\section{References}

1. Gibbs, P., et al., Comparison of quantitative T2 mapping and diffusion-weighted imaging in the normal and pathologic prostate. Magn Reson Med, 2001. 46(6): p. 1054-8.

2. deSouza, N.M., et al., Diffusion-weighted magnetic resonance imaging: a potential non-invasive marker of tumour aggressiveness in localized prostate cancer. Clin Radiol, 2008. 63(7): p. 774-82.

3. Shinmoto, H., et al., Biexponential apparent diffusion coefficients in prostate cancer. Magn Reson Imaging, 2009. 27(3): p. 355-9.

4. Langer, D.L., et al., Prostate tissue composition and MR measurements: investigating the relationships between $A D C, T 2, K($ trans $), v(e)$, and corresponding histologic features. Radiology, 2010. 255(2): p. 485-94.

5. Turkbey, B., et al., Is apparent diffusion coefficient associated with clinical risk scores for prostate cancers that are visible on 3-T MR images? Radiology, 2011. 258(2): p. 488-95.

6. Woodfield, C.A., et al., Diffusion-weighted MRI of peripheral zone prostate cancer: comparison of tumor apparent diffusion coefficient with Gleason score and percentage of tumor on core biopsy. AJR Am J Roentgenol, 2010. 194(4): p. W31622.

7. Hambrock, T., et al., Relationship between apparent diffusion coefficients at 3.0-T MR imaging and gleason grade in peripheral zone prostate cancer. Radiology, 2011. 259(2): p. 453-61.

8. Verma, S., et al., Assessment of aggressiveness of prostate cancer: correlation of apparent diffusion coefficient with histologic grade after radical prostatectomy. AJR Am J Roentgenol, 2011. 196(2): p. 374-81.

9. Rosenkrantz, A.B., et al., Prostate cancer: feasibility and preliminary experience of a diffusional kurtosis model for detection and assessment of aggressiveness of peripheral zone cancer. Radiology, 2012. 264(1): p. 126-35.

10. Panagiotaki, E., et al., Microstructural characterization of normal and malignant human prostate tissue with vascular, extracellular, and restricted diffusion for cytometry in tumours magnetic resonance imaging. Invest Radiol, 2015. 50(4): p. 218-27.

11. Gorelick, L., et al., Prostate histopathology: learning tissue component histograms for cancer detection and classification. Medical Imaging, IEEE Transactions on, 2013. 32(10): p. 1804-1818.

12. Zynger, D. and A. Parwani, Prostate Pathology. 2014: Demos Medical Publishing.

13. Mills, R., Self-Diffusion in Normal and Heavy-Water in Range 1-45 Degrees. Journal of Physical Chemistry, 1973. 77(5): p. 685-688.

14. Harris, K.R. and L.A. Woolf, Pressure and Temperature-Dependence of the SelfDiffusion Coefficient of Water and O-18 Water. Journal of the Chemical SocietyFaraday Transactions I, 1980. 76: p. 377-385.

15. Mulkern, R.V., et al., Biexponential characterization of prostate tissue water diffusion decay curves over an extended b-factor range. Magn Reson Imaging, 2006. 24(5): p. 563-8.

16. Storås, T.H., et al., Prostate magnetic resonance imaging: multiexponential T2 decay in prostate tissue. J Magn Reson Im 2008. 28(5): p. 1166-1172.

17. Gilani, N., et al., Minimization of errors in biexponential T2 measurements of the prostate. J Magn Reson Imaging, 2015. 42(4): p. 1072-7. 
18. Gilani, N., P. Malcolm, and G. Johnson, Decreases in the apparent diffusion coefficient of fast diffusing water in prostate cancer: A Monte Carlo study Submitted.

19. Papanikolaou, N., et al., Biexponential T2 relaxation time analysis of the brain: correlation with magnetization transfer ratio. Invest Radiol, 2002. 37(7): p. 363-7.

20. Steens, S.C., et al., Reproducibility of brain ADC histograms. Eur Radiol, 2004. 14(3): p. 425-30.

21. Fain, S., et al., Imaging of lung function using hyperpolarized helium-3 magnetic resonance imaging: Review of current and emerging translational methods and applications. J Magn Reson Imaging, 2010. 32(6): p. 1398-408.

22. Shemesh, N., et al., Detecting diffusion-diffraction patterns in size distribution phantoms using double-pulsed field gradient NMR: Theory and experiments. Journal of Chemical Physics, 2010. 132(3).

23. Sen, P.N., Time-dependent diffusion coefficient as a probe of geometry. Concepts in Magnetic Resonance Part A, 2004. 23A(1): p. 1-21.

24. Latour, L.L., et al., Pore-Size Distributions and Tortuosity in Heterogeneous PorousMedia. Journal of Magnetic Resonance Series A, 1995. 112(1): p. 83-91.

25. Jensen, J.H., et al., Diffusional kurtosis imaging: the quantification of non-gaussian water diffusion by means of magnetic resonance imaging. Magn Reson Med, 2005. 53(6): p. 1432-40.

26. Jensen, J.H. and J.A. Helpern, MRI quantification of non-Gaussian water diffusion by kurtosis analysis. NMR Biomed, 2010. 23(7): p. 698-710.

27. Codd, S.L. and P.T. Callaghan, Spin echo analysis of restricted diffusion under generalized gradient waveforms: planar, cylindrical, and spherical pores with wall relaxivity. Journal of Magnetic Resonance, 1999. 137(2): p. 358-372.

28. Callaghan, P.T., A simple matrix formalism for spin echo analysis of restricted diffusion under generalized gradient waveforms. Journal of Magnetic Resonance, 1997. 129(1): p. 74-84.

29. Callaghan, P.T., Pulsed-gradient spin-echo NMR for planar, cylindrical, and spherical pores under conditions of wall relaxation. Journal of Magnetic Resonance, Series A, 1995. 113(1): p. 53-59.

30. $\quad$ Price, W.S., NMR studies of translational motion: principles and applications. 2009: Cambridge University Press.

31. Fieremans, E., et al., Monte Carlo study of a two-compartment exchange model of diffusion. NMR in biomedicine, 2010. 23(7): p. 711-24.

32. Novikov, D.S., et al., Random walks with barriers. Nat Phys, 2011. 7(6): p. 508-514.

33. Doyle, S., et al., Cascaded discrimination of normal, abnormal, and confounder classes in histopathology: Gleason grading of prostate cancer. BMC bioinformatics, 2012. 13(1): p. 282.

34. Li, J.R., et al., Numerical simulation of diffusion MRI signals using an adaptive timestepping method. Phys Med Biol, 2014. 59(2): p. 441-54.

35. Regan, D.G. and P.W. Kuchel, Simulations of molecular diffusion in lattices of cells: insights for NMR of red blood cells. Biophysical journal, 2002. 83(1): p. 161-171.

36. Regan, D.G. and P.W. Kuchel, Mean residence time of molecules diffusing in a cell bounded by a semi-permeable membrane: Monte Carlo simulations and an expression relating membrane transition probability to permeability. European Biophysics Journal, 2000. 29(3): p. 221-227.

37. Lee, C.-Y., K.M. Bennett, and J.P. Debbins, Sensitivities of statistical distribution model and diffusion kurtosis model in varying microstructural environments: a Monte Carlo study. Journal of Magnetic Resonance, 2013. 230: p. 19-26. 
38. Einstein, A., Über die von der molekularkinetischen Theorie der Wärme geforderte Bewegung von in ruhenden Flüssigkeiten suspendierten Teilchen [On the Motion Required by the Molecular Kinetic Theory of Heat - of Small Particles Suspended in a Stationary Liquid]. Annalen der Physik, 1905. 322(8): p. 549-560.

39. Schneider, C.A., W.S. Rasband, and K.W. Eliceiri, NIH Image to ImageJ: 25 years of image analysis. Nature methods, 2012. 9(7): p. 671-675.

40. Xing, H., et al., Investigation of different boundary treatment methods in Monte-Carlo simulations of diffusion NMR. Magn Reson Med, 2013. 70(4): p. 1167-72.

41. Finkelstein, A., Water movement through lipid bilayers, pores, and plasma membranes. 1987: Wiley.

42. Regan, D.G. and P.W. Kuchel, Simulations of NMR-detected diffusion in suspensions of red cells: the effects of variation in membrane permeability and observation time. European Biophysics Journal, 2003. 32(8): p. 671-675.

43. Benga, G., et al., Comparative cell shape and diffusional water permeability of red blood cells from Indian elephant (Elephas maximus) and Man (Homo sapiens). Comparative Haematology International, 2000. 10(1): p. 1-8.

44. Haines, T. and L. Liebovitch, A molecular mechanism for the transport of water across phospholipid bilayers. Permeability and stability of lipid bilayers. CRC Press, Boca Raton, 1995: p. 123-136.

45. Stejskal, E.O. and J.E. Tanner, Spin diffusion measurements:spin echoes in the presence of a time-dependent field gradient. J. Chem. Phys., 1965. 42: p. 288-292.

46. Hall, M.G. and D.C. Alexander, Convergence and parameter choice for Monte-Carlo simulations of diffusion MRI. IEEE Trans Med Imaging, 2009. 28(9): p. 1354-64.

47. Pfeuffer, J., et al., Water signal attenuation in diffusion-weighted $1 H N M R$ experiments during cerebral ischemia: influence of intracellular restrictions, extracellular tortuosity, and exchange. Magn Reson Imaging, 1998. 16(9): p. 102332.

48. Stanisz, G.J., et al., An analytical model of restricted diffusion in bovine optic nerve. Magn Reson Med, 1997. 37(1): p. 103-11.

49. Herbst, M.D. and J. Goldstein, A review of water diffusion measurement by NMR in human red blood cells. American Journal of Physiology-Cell Physiology, 1989. 256(5): p. C1097-C1104.

50. Panagiotaki, E., et al., Microstructural Characterization of Normal and Malignant Human Prostate Tissue With Vascular, Extracellular, and Restricted Diffusion for Cytometry in Tumours Magnetic Resonance Imaging. signal, 2014. 9: p. 19-22.

51. Chatterjee, A., et al., Changes in epithelium, stroma, and lumen space predict ADC changes with prostate cancer Gleason grade. Proc Int Soc Magn Reson Med, 2014: p. 2155.

52. Bourne, R., et al., Effect of formalin fixation on biexponential modeling of diffusion decay in prostate tissue. Magn Reson Med, 2013. 70(4): p. 1160-6.

53. Reinoso, R.F., B.A. Telfer, and M. Rowland, Tissue water content in rats measured by desiccation. J Pharmacol Toxicol Methods, 1997. 38(2): p. 87-92.

54. Kiricuta, I.C., Jr. and V. Simplaceanu, Tissue water content and nuclear magnetic resonance in normal and tumor tissues. Cancer Res, 1975. 35(5): p. 1164-7.

55. Lu, H., et al., Three-dimensional characterization of non-Gaussian water diffusion in humans using diffusion kurtosis imaging (DKI). . NMR Biomed., 2006. 19: p. 236247.

56. Trampel, R., et al., Diffusional kurtosis imaging in the lung using hyperpolarized 3He. Magn Reson Med, 2006. 56(4): p. 733-7. 
57. Raab, P., et al., Cerebral gliomas: diffusional kurtosis imaging analysis of microstructural differences. Radiology, 2010. 254(3): p. 876-81.

58. Fieremans, E., J.H. Jensen, and J.A. Helpern, White matter characterization with diffusional kurtosis imaging. NeuroImage, 2011. 58(1): p. 177-88.

59. Anderson, S.W., et al., Characterizing non-gaussian, high b-value diffusion in liver fibrosis: Stretched exponential and diffusional kurtosis modeling. J Magn Reson Imaging, 2014. 39(4): p. 827-34.

60. Pentang, G., et al., Diffusion kurtosis imaging of the human kidney: a feasibility study. Magn Reson Imaging, 2014. 32(5): p. 413-20.

61. Hall, M.G., et al., Assessment of non-Gaussian diffusion with singly and doubly stretched biexponential models of diffusion-weighted MRI (DWI) signal attenuation in prostate tissue. NMR Biomed, 2015. 28(4): p. 486-95.

62. Chatterjee, A., et al., Changes in Epithelium, Stroma, and Lumen Space Correlate More Strongly with Gleason Pattern and Are Stronger Predictors of Prostate ADC Changes than Cellularity Metrics. Radiology, 2015. 277(3): p. 751-62.

63. Bennett, K.M., et al., Characterization of continuously distributed cortical water diffusion rates with a stretched-exponential model. Magn Reson Med, 2003. 50(4): p. 727-34.

64. Bourne, R.M., The trouble with apparent diffusion coefficient papers. Journal of Medical Radiation Sciences, 2015. 62(2): p. 89-91.

65. Bourne, R.M., et al., Biexponential diffusion decay in formalin-fixed prostate tissue: preliminary findings. Magn Reson Med, 2012. 68(3): p. 954-9. 